\title{
Designing a novel monitoring approach for the effects of space travel on astronauts' health
}

Anurag Sakharkar ${ }^{1}$ and Jian Yang, ${ }^{2 *}$

${ }^{1}$ College of Arts and Science, University of Saskatchewan, 9 Campus Drive, Saskatoon, SK S7N

5A5, Canada

${ }^{2}$ College of Pharmacy and Nutrition, University of Saskatchewan, 107 Wiggins Road, Saskatoon, SK S7N 5E5, Canada

*Corresponding author. Tel: 1-306-966-6361; Email: jian.yang@usask.ca 


\begin{abstract}
Space exploration and extraterrestrial civilization have fascinated humankind since the earliest days of human history. However, it was only until last century that humankind finally began taking significant steps towards these goals by sending astronauts into space, landing on the moon, and building the International Space Station. However, space voyage is very challenging and dangerous, and astronauts are under constant space radiation and microgravity. It has been shown that astronauts are at a high risk of developing a broad range of diseases/disorders. Thus, it is critical to develop a rapid and effective assay to monitor astronauts' health in space. In this study, gene expression and correlation patterns were analyzed for 10 astronauts (8 male and 2 female) using the publicly available microarray dataset E-GEOD-74708. We identified 218 differentially expressed genes between In-flight and Pre-flight and noticed that space travel decreased genome regulation and gene correlations across the entire genome, as well as individual signaling pathways. Furthermore, we rationally designed a rapid assay of 32 genes which could be used to monitor astronauts' health during space travel. Further studies, including microgravity experiments, are warranted to optimize and validate the proposed assay.
\end{abstract}




\section{Introduction}

"Space: the final frontier. These are the voyages of the starship Enterprise. Its continuing mission: to explore strange new worlds. To seek out new life and new civilizations. To boldly go where no one has gone before!"'. Every fan of the American science fiction TV series Star Trek is likely to remember this opening monologue. Indeed, space exploration and extraterrestrial civilization have fascinated humankind since the early days. However, it was only until last century that humankind finally took a solid step forward by sending astronauts into space and landing on the moon ${ }^{2,3}$. Into the $21^{\text {st }}$ century, humankind has made another great leap forward in space exploration, expediting the International Space Station (ISS) programme ${ }^{4}$, sending unmanned rovers to seek signs of life on Mars ${ }^{5,6}$, and starting private space ventures ${ }^{7,8}$. In addition, NASA (The National Aeronautics and Space Administration) is planning to send astronauts back to the moon in 2024 and to Mars in the 2030s $\mathrm{s}^{9}$. Through all these endeavors, we have seen the twilight of manned space missions going beyond our harbor (Earth) and into deep space.

Space travel is challenging, dangerous and full of uncertainty. Astronauts are under sustained microgravity and cosmic radiation. Prolonged exposure to microgravity and cosmic radiation has been shown to cause a series of health problems, including loss of muscle mass ${ }^{10,11}$, reduced bone density $^{12,13}$, compromised immune responses ${ }^{14,15}$, impaired renal functions ${ }^{16}$, neurological system irresponsiveness ${ }^{17,18}$, and development of cardiovascular diseases ${ }^{19,20}$. Furthermore, microgravity and cosmic radiation can cause several types of cancer, such as leukemia, likely due to compromised immunity ${ }^{21}$. However, studies under simulated microgravity also show that microgravity affects cell proliferation and apoptosis and can change cancer cells into less malignant phenotypes ${ }^{22-24}$. These studies indicate that the impact of microgravity and cosmic radiation on human health is profound, and it is unlikely that this impact is due to changes in one 
gene or a small group of genes. Therefore, genomic, transcriptomic and/or proteomic studies are crucial to fully understand the effects of microgravity and cosmic radiation on human health. These studies can lead to the development of effective strategies to monitor, prevent, and/or alleviate the effects on human health caused by space travel.

Approximately 12 years ago, NASA and JAXA (Japan Aerospace Exploration Agency) undertook a microarray study on 10 astronauts ( 8 male and 2 female), who had a six-month mission at the ISS ${ }^{25}$. Terada et al. used qPCR to confirm that space flight changed gene expression in astronauts $^{25}$. Several genes, including FGF18, ANGPTL7 and COMP, were upregulated and might play a role in inhibiting cell proliferation in hair follicles. However, they neither discussed the potential effects of the differentially expressed genes on the astronauts' health nor provided any strategy to monitor these effects. Thus, in the current study, we reanalyzed this microarray dataset, rationally developed a method which could monitor the effects of space travel on astronauts' health and searched for potential intervention options.

\section{Results and discussion}

\section{Differentially expressed genes (DEGs)}

NASA and JAXA undertook Study GLDS-174: "Effects of a closed space environment on gene expression in hair follicles of astronauts in the International Space Station” between 2009 and 2013. However, Terada et al. only reported the expression of selected genes in this study and did not undertake further analysis ${ }^{25}$. To better understand how space travel affects astronauts' health, we downloaded the microarray dataset (E-GEOD-74708) from the NASA GeneLab open data repository. The microarray dataset includes gene expression information for three stages: Preflight (6 months to 2 weeks before launch), In-flight (while staying in the ISS), and Post-flight (2 days to 3 months after returning from the ISS). We then analyzed the dataset for gene expression 
and gene pair correlation. Using cut-off criteria of $\left|\log _{2} \mathrm{FC}\right| \geq 3.00$ and $p<0.05,218$ DEGs were identified between In-flight and Pre-flight (Supplementary File 1: DEG_list.xls). The top 20 upand down-regulated genes are summarized in Table 1. However, no DEGs were detected between Post-flight and In-flight, suggesting that the astronauts may need a longer time than current guidelines for the body to adjust to the ground environment. Additionally, astronauts have a much higher risk of developing various types of diseases than normal people and precautionary measures are critical to protect their health after retuning to ground.

As expected, the DEGs between In-flight and Pre-flight regulate a broad spectrum of biological and physiological functions. For example, the most up-regulated gene LIMCH1, encoding LIM and calponin-homology domains 1, activates the non-muscle myosin IIa complex, stabilizes focal adhesion, and inhibits cell migration ${ }^{26,27}$. Thus, the upregulation of LIMCH1 may explain observations that microgravity exposure can change cancer cells into less malignant phenotypes $^{22-24}$. The second most up-regulated gene IER3 encodes Immediate Early Response 3 and regulates cell apoptosis ${ }^{28}$, imflammation ${ }^{29}$ and tumorigenesis ${ }^{30}$. Upregulation of $I E R 3$ has been observed in different types of cancer and may regulate cancer progression ${ }^{31,32}$. This implies another possible reason, other than radiation exposure, which increases the risk of developing cancer in astronauts during a long period of space travel. The most down-regulated gene, NFATC1, encodes Nuclear Factor of Activated T Cells 1 and plays important roles in osteoblast differentiation ${ }^{33}$, osteoclastogenesis $^{34}$, T-cell differentiation ${ }^{35}$, lymphatic endothelial development ${ }^{36}$, cardiac valve morphogenesis $^{37}$ and tumorigenesis ${ }^{38}$. The downregulation of NFATC1 may result in osteoporosis, immunocompromised conditions, and prostate cancer in astronauts during a prolonged space stay. The second most down-regulated gene, KIDINS220, encoding Kinase D Interacting Substrate 220, modulates the development and function of the nerve and cardiovascular systems ${ }^{39,40}$. The 
downregulation of KIDINS220 may contribute to the development of neurological disorders and cardiovascular diseases in astronauts. In summary, the large variation of the biological processes regulated by the DEGs provides a valuable resource of genes for us to develop a rapid assay kit ( 20-30 genes) to monitor astronauts’ health conditions in space. However, it is noteworthy that the three genes reported by Terada et al., FGF18, ANGPTL7 and COMP, are not in the aforementioned DEG list as they failed to meet the cut-off criteria.

\section{Gene pair correlations}

As mentioned above, the effect of space travel on astronaut's health is comprehensive and involves the regulation of many genes. It is also well-known that any biological, physiological, or pathophysiological change requires a delicate coordination of multiple genes. Thus, we decided to carry out a gene pair correlation analysis for the whole genome (30645 transcripts for 23115 genes) among the Pre-flight, In-flight, and Post-flight expression profiles. As shown in Figure 1A, the genes are highly correlated in the Pre-flight, which This is consistent with previous studies showing that gene expressions are correlated in normal human tissues ${ }^{41}$. Upon staying in the ISS, the genome appeared to significantly lose its regulation of the gene expression and gene coordination was scrambled (Figure 1B). Our previous studies have shown that loss of gene pair correlations is a hallmark of carcinogenesis and/or cancer progression ${ }^{42-44}$. Therefore, loss of coordination of gene expression, other than cosmic radiation and compromised immune system, is likely to be another factor which increases the risk of cancer development in astronauts. Analysis of the Post-flight dataset showed that returning to ground did not significantly improve genome regulation and coordination of gene expression (Figure 1C), implicating that the effect of space travel on human health is more profound and longer lasting than currently thought. Extended care is necessary for astronauts to minimize the risk of disease development. As we progress towards 
manned missions into outer space (for example, to the Moon and Mars) and other prolonged space stays, it is critical to develop rapid approaches to monitor genome regulation and establish corresponding protocols to alleviate the effects of space travel on human health. Another important question that needs to be addressed is whether the human body could establish a new "routine" for prolonged space stays and what this would look like in terms of astronauts' health. We speculate that loss of gene expression coordination might also be responsible for other diseases/conditions astronauts experience, such as muscle loss and compromised immune system. Extensive further studies in astrobiology, astromedicine and astropharmacy are warranted to prepare us for longerterm outer space exploration.

\section{Signaling pathway and disease network of the DEGs}

Since DEGs are the most altered genes during a biological process, we conducted a signaling pathway analysis of the 218 DEGs between In-flight and Pre-flight to figure out what biological functions are regulated by these genes. The top 11 signaling pathways were identified to be signal transduction (16 genes: ARHGAP9, CCL2, CCNC, CHN1, CLTB, COLAA4, CREB1, CRHR1, CTNNBIP1, HIF1A, KIDINS220, NFATC1, PDPK1, SOS2, THBS3 and YES1), immune system (11 genes: ATF2, BIRC2, CREB1, EIF4E2, IL7, NFATC1, PDPK1, UBA5, UBR4, XAF1 and YES1), gene expression (10 genes: AARS2, CCNC, RRN3, ZNF184, ZNF253, ZNF529, ZNF606, ZNF664, ZNF699 and ZNF711), metabolism (10 genes: ACSL4, ARSK, CCNC, GM2A, GPT, HACL1, NDUFA1, PIKFYVE and PSAT1), metabolism of proteins (9 genes: ARSK, CCL2, DPP4, GNE, MAGT1, PCSK1, SPON2 and XRN2), generic transcription pathway (8 genes: CCNC, ZNF184, ZNF253, ZNF529, ZNF606, ZNF664, ZNF699 and ZNF711), developmental biology (7 genes: CCNC, CLTB, COLAA4, CREB1, SCN2B, SOS2 and YES1), metabolism of lipids and lipoproteins (6 genes: ACSL4, ARSK, CCNC, GM2A, HACL1 and PIKFYVE), axon guidance (6 
genes: $C L T B, C O L A A 4, C R E B 1, S C N 2 B, S O S 2$ and $Y E S 1)$, innate immune system (6 genes: ATF2, BIRC2, CREB1, NFATC1, PDPK1 and YES1) and disease (6 genes: CCNC, CHMP4C, CREB1, CTNNBIP1, HIF1A and PDPK1). In total, 48 genes were found to be involved in the regulation of these top 11 signaling pathways. Moreover, it is obvious that most of these DEGs are involved in the regulation of multiple signaling pathways, and that these signaling pathways regulate a broad range of biological, physiological and/or pathophysiological processes. This analysis reconfirms that space travel imposes comprehensive effects on the human system rather than affecting an individual organ or tissue.

We further analyzed the disease network for the 218 DEGs between the In-flight and Preflight expression profiles to identify the major disease/disorder conditions associated with space travel (Supplementary File 2: Disease_DEG.xls). As shown in Table 2, the top 20 disease/disorder conditions can broadly be divided into three categories: neoplasia/carcinoma, neurological disorder, and liver function, with the top 3 conditions being malignant neoplasm of the breast, colorectal carcinoma and malignant neoplasm of the prostate. More DEGs were associated with tumor development than other diseases/disorders. In 2019, Reynolds et al. reported the effect of space radiation on astronauts' death using a statistical analysis ${ }^{45}$. Of the astronauts and cosmonauts who traveled to space between 1960 and 2018, 53 NASA astronauts have died, with 16 of those deaths $(30.2 \%)$ being caused by cancer. Nevertheless, their study indicates that space radiation does not have a strong impact on the mortality of astronauts. This implies that the weakened genome regulation (i.e., reduced gene expression correlation) we observed in this study, other than compromised immune function, is likely to play an important role in tumor development in astronauts, even though the tumors may be less malignant or even benign. Moreover, consistent with previous studies ${ }^{46,47}$, our results showed that space travel significantly affects astronauts' liver 
function and liver metabolism. Key genes identified from our analysis, such as GPT and UBA5, might serve as valuable monitoring biomarkers of astronauts' liver function and even possible medical intervention points to protect astronauts' health. Since liver is a major organ for drug metabolism, a systematic astropharmacy study is needed to establish drug profiles, including dosing, ADMET (absorption, distribution, metabolism, excretion, and toxicity) and even formulation, in preparation for future voyages into outer space. Space drug usage based on current information may be less effective or even detrimental to astronauts' health.

\section{Design of a rapid assay for space travel}

Any biological or physiological process requires a delicate regulation of genes, including gene expression levels, interactions, and correlations. However, most genomics/genetic studies are focused on gene expression level rather than gene pair correlation. Although gene co-expressions and protein co-localizations are commonly studied in biomedical research, our research laboratory, to the best of our knowledge, is the first to apply gene pair correlation coefficient (a mathematical term) to describe and explain biological and medical questions ${ }^{42-44}$. Thus, in order to design a rapid assay to monitor astronauts' health, we decided to adopt a set of genes with not only expression levels but also gene pair correlations significantly altered by space travel.

We calculated gene pair correlation coefficients (designated as CC) of the DEGs associated with the top 11 signaling pathways for the Pre-flight, In-flight and Post-flight datasets, and analyzed the gene pair correlation coefficient differentials (designated as $\Delta \mathrm{CC}$ ) between the Inflight and Pre-flight and between the Post-flight and In-flight (Figure 2). The changes in gene pair CCs between the In-flight and Pre-flight datasets were obvious for every single signaling pathway except the generic transcription pathway. However, the changes between Post-flight and In-flight CCs were minimal. Thus, using $|\Delta \mathrm{CC}|>0.70$ as a cut-off, we identified gene pairs with their 
correlation coefficients significantly altered between the In-flight and Pre-flight datasets and summarized them in Table 3. These gene pairs were then classified into four categories based on changes in correlation coefficients: Category 1 - positive to more/less positive (highlighted in blue), Category 2 - positive to negative (highlighted in green), Category 3 - negative to positive (highlighted in brown), and Category 4 - negative to more/less negative (highlighted in red). It is noteworthy that Categories 2 and 3 contained more gene pairs than Categories 1 and 4, implying that many genes lost their coordination and even started counteractive processes in space. This observation supports previous studies that spaceflight significantly affects gene expression and homeostasis $^{48}$. Apparently, this dramatic change in gene expression coordination affects normal biological and physiological functions in the human body and is likely detrimental to astronauts' health.

In a previous study by Taylor ${ }^{49}$, correlation was classified into three categories based on correlation coefficients: strong $(0.68 \leq \mathrm{CC} \leq 1.00)$, moderate $(0.36 \leq \mathrm{CC} \leq 0.67)$ and weak $(\mathrm{CC} \leq$ 0.35). To increase the sensitivity of the assay for space travel, it is rational to only include gene pairs with correlation changing from strong to weak, weak to strong, or strong to opposite strong besides $|\Delta C C|>0.70$. Using this guideline, we identified the following gene pairs; 12 gene pairs (CCL2 - COL4A4, CCL2 - CREB1, CCL2 - CRHR1,CCL2 - THBS3, COLAA4 - CREB1, CREB1 - CRHR1, CREB1 - THBS3, CTNNBIP1 - NFATC1, KIDINS220 - PDPK1, KIDINS220 - SOS2, KIDINS220 - YES1, and NFATC1 - THBS3) from signal transduction, 11 gene pairs (ATF2 NFATC1, BIRC2 - PDPK1, CREB1 - EIF4E2, CREB1 - IL7, CREB1 - UBR4, CREB1 - XAF1, EIF4E2 - IL7, EIF4E2 - XAF1,IL7 - UBR4, NFATC1 - UBR4, and UBR4 - XAF1) from immune system, 2 gene pairs (AARS2 - ZNF606 and RRN3 - ZNF606) from gene expression, 5 gene pairs (ACSL4 - CCNC, ACSL4 - NDUFA1, ACSL4 - PIKFYVE, CCNC-PSAT1 and GPT-PIKFYVE) 
from metabolism, 6 gene pairs (CCL2 - DPP4, CCL2 - PCSK1, CCL2 - SPON2, DPP4 - GNE, MAGT1 - PCSK1 and PCSK1 - SPON2) from metabolism of proteins, 1 gene pair (COLAA4 CREB1) from developmental biology, 2 gene pairs (ACSL4 - CCNC and ACSL4 - PIKFYVE) from metabolism of lipids and lipoprotein, 1 gene pair (COL4A4 - CREB1) from axon guidance, 2 gene pairs $(A T F 2$ - NFATC1 and BIRC2 - PDPK1) from innate immune system, and 1 gene pair $(C H M P 4 C-C R E B 1)$ from disease. In total, 32 genes were selected, and they are AARS2, ACSL4, ATF2, BIRC2, CCL2, CCNC, CHMP4C, COLAA4, CREB1, CRHR1, CTNNBIP1, DPP4, EIF4E2, GNE, GPT, IL7, KIDINS220, MAGT1, NDUFA1, NFATC1, PCSK1, PDPK1, PIKFYVE, PSAT1, RRN3, SOS2, SPON2, THBS3, UBR4, XAF1, YES1, and ZNF606. To get a global view of these genes in regulating biological functions, we constructed the protein-protein interaction (PPI), disease-gene, drug-gene and miRNA-gene networks for the 218 DEGs and labelled the 32 genes in the networks (Figure 3). Genes CCNC, CTNNBIP1, EIF4E2 and RRN3 are not present in the disease-gene network; genes BIRC2, CCNC, CHMP4C, CTNNBIP1, EIF4E2, IL7, KIDINS220, MAGT1, PIKFYVE, RRN3, SOS2, SPON2, THBS3, XAF1 and ZNF606 are not present in the druggene network; and genes CRHR1, DPP4, GPT and SPON2 are not present in the miRNA-gene network, respectively. Most of the 32 identified genes have high degrees of connectivity and are likely to be biological hubs. Thus, we propose that an $\mathrm{RT}^{2}$-PCR assay of these 32 genes could be a novel, effective and comparatively cheap way to continuously monitor astronauts' health in space. Validation of this assay under microgravity simulation conditions is warranted for further optimization of the gene list before sending an assay kit for practical tests in space. Finally, we extracted the drug molecules (both approved and experimental, listed in Supplementary File 3: Drug_list.xls) and miRNAs (listed in Supplementary File 4: miRNA_list.xls), which potentially target these 32 genes from the drug-gene and miRNA-gene networks. These drugs and miRNAs 
could be applied as possible medical interventions for health conditions associated with space travel.

\section{Methods}

\section{Data acquisition}

Microarray dataset E-GEOD-74708, which is the largest publicly available space gene expression dataset, was downloaded from the NASA GeneLab Database (https:/genelabdata.ndc.nasa.gov/genelab). It contains gene expression profiles for 10 astronauts (8 male and 2 female), who stayed in the ISS between July 2009 and February 2013. For each astronaut, 2 profiles were collected at each of the three time points, Pre-flight (prior to departing for the ISS), In-flight (while staying at the ISS) and Post-flight (after return to the ground).

\section{Data processing}

Individual gene expression profiles were combined into three DataFrames: Pre-flight, Inflight, and Post-flight using the Pandas package (version 1.3.0) ${ }^{50}$ in Python. These DataFrames were analyzed for possible outliers and verified for accuracy. Gene expression readings with null values were removed. Then, Agilent IDs in the data were mapped to gene names using the Ensembl BioMart database (https://www.ensembl.org/biomart/martview).

DEG identification and signaling pathway analysis

Differentially expressed genes (DEGs: $\left|\log _{2} \mathrm{FC}\right| \geq 3.00$ and $p<0.05$ ) were identified between the In-flight and the Pre-flight DataFrames using the DEGseq package (version 1.42.0) in $\mathrm{R}^{51}$. The Likelihood Ratio Test (LRT) function was applied in the calculation. No DEGs were identified between the Post-flight and the In-flight DataFrames. The DEGs between In-flight and Pre-flight were then subjected to signaling pathway analysis using the InnateDB tool ${ }^{52}$.

\section{Calculation of gene pair correlation matrices}


Pairwise Pearson correlation coefficient matrices for the 3 gene expression DataFrames were calculated using formula $r=\frac{\Sigma\left(x_{i}-\underline{x}\right)\left(y_{i}-\underline{y}\right)}{\sqrt{\Sigma\left(x_{i}-\underline{x}\right)^{2} \Sigma\left(y_{i}-\underline{y}\right)^{2}}}$. The correlation matrices were subsequently transferred to be visualized using an R platform. To analyze gene pair correlations for the signaling pathways, the expression profiles of the genes for the pathways were extracted from the DataFrames to produce the DataFrame subsets. For each signalling pathway, pairwise Pearson correlation coefficients ware calculated and visualized as outlined above between the In-flight and Pre-flight subsets and between the Post-flight and In-flight subsets. Positive and negative correlations were represented in blue and red, respectively. Additionally, gene pair correlation coefficient differentials, $\Delta \mathrm{CC}($ In-Pre $)=\mathrm{CC}_{\text {In-flight }}-\mathrm{CC}_{\text {Pre-flight }}$ and $\Delta \mathrm{CC}($ Post-In $)=\mathrm{CC}_{\text {Post-flight }}-$ $\mathrm{CC}_{\text {In-flight }}$ were calculated by matrix subtraction between the In-flight and Pre-flight subsets and between the Post-flight and In-flight subsets. These were subsequently visualized with correlation difference plots with positive and negative $\Delta \mathrm{CC}$ s shown in green and red, respectively.

\section{Protein-protein interaction network and disease network constructions}

The BioGrid database ${ }^{53}$ and DisGeNET database ${ }^{54}$ were downloaded in March 2021 to create a protein-protein interaction (PPI) network (Figure 3A) and extract the disease interaction information (Figure 3B), respectively, for the DEGs. The networks were then constructed using Python and visualized using Cytoscape ${ }^{55}$.

\section{Drug and miRNA screenings}

The Drugbank $^{56}$ and miRTARBase ${ }^{57}$ databases were also downloaded in March 2021 to extract the drug (Figure 3C) and miRNA (Figure 3D) interaction information for the DEGs. The networks were subsequently constructed using Python and visualized using Cytoscape ${ }^{55}$. From these two networks, we identified potential drugs and miRNAs targeting the 32 genes we identified 
to be used in a rapid $\mathrm{RT}^{2}$-PCR assay for space travel (Supplementary File 3: Drug_list.xls and Supplementary File 4: miRNA_list.xls).

\section{Conclusion}

In this study, we analyzed the effects of space travel on gene expression and correlation using the publicly available microarray dataset E-GEOD-74708 and systematically developed a novel rapid assay which could be used to monitor astronauts' health in space. However, this study faces several limitations. First, the sample size (10 astronauts) is small due to the nature of space travel and unstandardized astrobiological research. More sample collection is needed to optimize the monitoring assay. Secondly, there were only 2 female astronauts in the microarray dataset. To get better representation and more reliable accuracy in the analysis, more gene expression profiles from a diverse population of astronauts should be obtained. Thirdly, our laboratory is not equipped with any microgravity infrastructure, and thus, we cannot validate the proposed assay. However, in our future studies, we will reach out to resources such as microgravity simulators and continue to conduct research in optimizing and validating this assay. Despite these drawbacks, our current study proposes a new strategy to develop genome-based rapid assays. This approach could also be applied to other research fields, such as cancer diagnostic assays. 


\section{Funding}

None

\section{Conflict of interest}

The authors declare that no conflict of interest exists.

\section{Author contribution}

This research was originated by J.Y. and carried out by A.S. and J.Y. 


\section{References}

1. Roddenberry, G. Star Trek: The Next Generation 1, (1987).

2. Cole, M. D. Vostok 1: First human in space. (Enslow Publishers, 1995).

3. Loff, S. Apollo 11 Mission Overview. Apollo $11 \mid$ NASA (2015). Available at: https://www.nasa.gov/mission_pages/apollo/missions/apollo11.html.

4. Garcia, M. International Space Station. International Space Station | NASA (2015). Available at: https://www.nasa.gov/mission_pages/station/main/.

5. Mars Exploration Program. Mars Curiosity Rover. Curiosity Rover | NASA (2021). Available at: https://mars.nasa.gov/msl/.

6. Greicius, T. Mars Perseverance Rover. Perseverance Rover|NASA (2016). Available at: https://www.nasa.gov/perseverance.

7. Loff, S. Commercial Space Transportation. Commercial Space Economy | NASA (2015). Available at: https://www.nasa.gov/exploration/commercial/.

8. Guzman, A. Low-earth orbit economy. Low-Orbit Earth Economy | NASA (2021). Available at: https://www.nasa.gov/leo-economy/.

9. Mars, K. Gateway. Gateway|NASA (2019). Available at: https://www.nasa.gov/gateway.

10. Gopalakrishnan, R. et al. Muscle Volume, strength, endurance, and exercise loads during 6-month missions in Space. Aviation, Space, and Environmental Medicine 81, 91-104 (2010).

11. Trappe, S. et al. Exercise in space: Human skeletal muscle after 6 months aboard the International Space Station. Journal of Applied Physiology 106, 1159-1168 (2009).

12. Vogel, J. M. \& Whittle, M. W. Proceedings: Bone mineral content changes in the Skylab astronauts. American Journal of Roentgenology 126, 1296-1297 (1976).

13. Vico, L. \& Hargens, A. Skeletal changes during and after spaceflight. Nature Reviews Rheumatology 14, 229-245 (2018).

14. Akiyama, T. et al. How does spaceflight affect the acquired immune system? npj Microgravity 6, (2020).

15. Crucian, B. E. et al. Immune system dysregulation during spaceflight: Potential countermeasures for deep space exploration missions. Frontiers in Immunology 9, (2018).

16. Smith, S. M. et al. Bone metabolism and renal stone risk during International Space Station Missions. Bone 81, 712-720 (2015).

17. Roy-O'Reilly, M., Mulavara, A. \& Williams, T. A review of alterations to the brain during spaceflight and the potential relevance to crew in long-duration space exploration. $n p j$ Microgravity 7, (2021).

18. Kalb, R. \& Solomon, D. Space exploration, Mars, and the nervous system. Archives of Neurology 64, 485 (2007).

19. Vernice, N. A., Meydan, C., Afshinnekoo, E. \& Mason, C. E. Long-term spaceflight and the cardiovascular system. Precision Clinical Medicine 3, 284-291 (2020).

20. Hughson, R. L., Helm, A. \& Durante, M. Heart in space: Effect of the extraterrestrial environment on the cardiovascular system. Nature Reviews Cardiology 15, 167-180 (2017).

21. Cucinotta, F. A. et al. Space radiation cancer risks and uncertainties for Mars missions. Radiation Research 156, 682-688 (2001). 
22. Ahn, C. B. et al. Simulated microgravity with floating environment promotes migration of non-small cell lung cancers. Scientific Reports 9, (2019).

23. Lewis, M. L. et al. Spaceflight alters microtubules and increases apoptosis in human lymphocytes (Jurkat). The FASEB Journal 12, 1007-1018 (1998).

24. Krüger, M. et al. Fighting thyroid cancer with microgravity research. International Journal of Molecular Sciences 20, 2553 (2019).

25. Terada, M. et al. Effects of a closed space environment on gene expression in hair follicles of astronauts in the International Space Station. PLOS ONE 11, (2016).

26. Lin, Y.-H. et al. Limch1 regulates nonmuscle myosin-II activity and suppresses cell migration. Molecular Biology of the Cell 28, 1054-1065 (2017).

27. Aguilar-Cuenca, R., Juanes-García, A. \& Vicente-Manzanares, M. Myosin II in mechanotransduction: Master and commander of cell migration, morphogenesis, and cancer. Cellular and Molecular Life Sciences 71, 479-492 (2013).

28. Schilling, D., Pittelkow, M. R. \& Kumar, R. IEX-1, an immediate early gene, increases the rate of apoptosis in keratinocytes. Oncogene 20, 7992-7997 (2001).

29. Arlt, A. \& Schäfer, H. Role of the immediate early response 3 (IER3) gene in cellular stress response, inflammation and tumorigenesis. European Journal of Cell Biology 90, 545-552 (2011).

30. Ustyugova, I. V., Zhi, L., Abramowitz, J., Birnbaumer, L. \& Wu, M. X. IEX-1 deficiency protects against Colonic Cancer. Molecular Cancer Research 10, 760-767 (2012).

31. Akilov, O. E., Wu, M. X., Ustyugova, I. V., Falo, L. D. \& Geskin, L. J. Resistance of sézary cells to TNF- $\alpha$-induced apoptosis is mediated in part by a loss of TNFR 1 and a high level of the IER3 expression. Experimental Dermatology 21, 287-292 (2012).

32. Rasmussen, L. M. et al. Prolactin and oestrogen synergistically regulate gene expression and proliferation of breast cancer cells. Endocrine-Related Cancer 17, 809-822 (2010).

33. Bergamin, L. S. et al. Expression and function of the P2X7 receptor in human osteoblasts: The role of NFATC1 transcription factor. Journal of Cellular Physiology 236, 641-652 (2020).

34. Winslow, M. M. et al. Calcineurin/NFAT signaling in osteoblasts regulates bone mass. Developmental Cell 10, 771-782 (2006).

35. Klein-Hessling, S. et al. NFATC1 controls the cytotoxicity of CD8+ T cells. Nature Communications 8, (2017).

36. Kulkarni, R. M., Greenberg, J. M. \& Akeson, A. L. NFATC1 regulates lymphatic endothelial development. Mechanisms of Development 126, 350-365 (2009).

37. Chang, C.-P. et al. A field of myocardial-endocardial NFAT signaling underlies heart valve morphogenesis. Cell 118, 649-663 (2004).

38. Xu, W. et al. NFATC1 promotes cell growth and tumorigenesis in ovarian cancer upregulating C-myc through ERK1/2/p38 MAPK signal pathway. Tumor Biology 37, 44934500 (2015).

39. Cesca, F. et al. KIDINS220/arms mediates the integration of the neurotrophin and VEGF pathways in the vascular and nervous systems. Cell Death \& Differentiation 19, 194-208 (2011).

40. Cesca, F. et al. KIDINS220/arms is an essential modulator of Cardiovascular and nervous system development. Cell Death \& Disease 2, (2011). 
41. van Dam, S., Võsa, U., van der Graaf, A., Franke, L. \& de Magalhães, J. P. Gene coexpression analysis for functional classification and gene-disease predictions. Briefings in Bioinformatics (2017). doi:10.1093/bib/bbw139

42. Sakharkar, M. K., Rajamanickam, K., Ji, S., Dhillon, S. K. \& Yang, J. Pairwise correlation of genes involved in glucose metabolism: A potential diagnostic marker of cancer? Genes \& Cancer 12, 69-76 (2021).

43. Sakharkar, M. K., Kaur Dhillon, S., Chidambaram, S. B., Essa, M. M. \& Yang, J. Gene pair correlation coefficients in sphingolipid metabolic pathway as a potential prognostic biomarker for breast cancer. Cancers 12, 1747 (2020).

44. Sakharkar, M. K. et al. Alteration in gene pair correlations in tryptophan metabolism as a hallmark in cancer diagnosis. International Journal of Tryptophan Research 13, 117864692097701 (2020).

45. Reynolds, R. J. et al. Contrapositive logic suggests space radiation not having a strong impact on mortality of US astronauts and Soviet and Russian cosmonauts. Scientific Reports 9, (2019).

46. Beheshti, A. et al. Multi-omics analysis of multiple missions to space reveal a theme of lipid dysregulation in mouse liver. Scientific Reports 9, (2019).

47. Kurosawa, R. et al. Impact of spaceflight and artificial gravity on sulfur metabolism in mouse liver: Sulfur Metabolomic and transcriptomic analysis. Scientific Reports 11, (2021).

48. Garrett-Bakelman, F. E. et al. The NASA twins study: A multidimensional analysis of a year-long human spaceflight. Science 364, (2019).

49. Taylor, R. Interpretation of the correlation coefficient: A basic review. Journal of Diagnostic Medical Sonography 6, 35-39 (1990).

50. McKinney, W. Data Structures for Statistical Computing in python. Proceedings of the Python in Science Conference (2010). doi:10.25080/majora-92bf1922-00a

51. Wang, L., Feng, Z., Wang, X., Wang, X. \& Zhang, X. DEGseq: An R package for identifying differentially expressed genes from RNA-Seq Data. Bioinformatics 26, 136138 (2009).

52. Lynn, D. J. et al. InnateDB: Facilitating systems-level analyses of the mammalian innate immune response. Molecular Systems Biology 4, 218 (2008).

53. Stark, C. BioGRID: A general repository for interaction datasets. Nucleic Acids Research 34, (2006).

54. Piñero, J. et al. The DisGeNET Knowledge Platform for Disease Genomics: 2019 update. Nucleic Acids Research (2019). doi:10.1093/nar/gkz1021

55. Shannon, P. et al. Cytoscape: A software environment for integrated models of Biomolecular Interaction Networks. Genome Research 13, 2498-2504 (2003).

56. Wishart, D. S. DrugBank: A comprehensive resource for in silico drug discovery and exploration. Nucleic Acids Research 34, (2006).

57. Huang, H.-Y. et al. MiRTarBase 2020: Updates to the experimentally validated micrornatarget interaction database. Nucleic Acids Research (2019). doi:10.1093/nar/gkz896 
Table 1. Top 20 up- and down-regulated DEGs (differentially expressed genes) between In-flight and Pre-flight. The cut-off criteria for DEGs are $\left|\log _{2} \mathrm{FC}\right| \geq 3.00$ and $p<0.05$.

\begin{tabular}{|l|r|r|l|r|r|}
\hline \multicolumn{3}{|c|}{ UP-REGULATED GENES } & \multicolumn{2}{c|}{ DOWN-REGULATED GENES } \\
\hline Gene Name & Log2 2 C & $\boldsymbol{p}$ Value & Gene Name & \multicolumn{1}{c|}{ Log2FC } & $\boldsymbol{p}$ Value \\
\hline LIMCH1 & 7.59 & 0.03 & NFATC1 & -10.40 & 0.00 \\
\hline IER3 & 7.45 & 0.03 & KIDINS220 & -10.35 & 0.03 \\
\hline ZNF664 & 6.57 & 0.03 & ZCCHC9 & -7.82 & 0.00 \\
\hline NDUFA1 & 6.27 & 0.03 & IGSF9 & -6.08 & 0.03 \\
\hline AL391650.1 & 5.28 & 0.03 & HSD11B1L & -5.76 & 0.00 \\
\hline TUBGCP5 & 5.07 & 0.03 & CLTB & -5.69 & 0.00 \\
\hline GAPVD1 & 5.03 & 0.03 & YIPF2 & -5.68 & 0.03 \\
\hline AARS2 & 4.81 & 0.03 & BTBD9 & -5.49 & 0.00 \\
\hline ACO04080.5 & 4.77 & 0.03 & LINC00668 & -5.41 & 0.00 \\
\hline NAA60 & 4.72 & 0.03 & AL096711.2 & -4.97 & 0.03 \\
\hline IGBP1P1 & 4.41 & 0.03 & EIF4E2 & -4.97 & 0.03 \\
\hline FANCD2 & 4.28 & 0.03 & SHE & -4.41 & 0.03 \\
\hline AMT & 4.20 & 0.03 & HOXC4 & -4.27 & 0.00 \\
\hline PNPLA4 & 4.11 & 0.03 & THBS3 & -4.17 & 0.00 \\
\hline RABGAP1 & 4.10 & 0.03 & PGM2L1 & -3.95 & 0.00 \\
\hline MAPKAPK5 & 3.89 & 0.03 & TCEANC & -3.93 & 0.03 \\
\hline DFFBP1 & 3.83 & 0.03 & ARHGAP9 & -3.91 & 0.00 \\
\hline STAG1 & 3.71 & 0.03 & ZNF451 & -3.80 & 0.03 \\
\hline SPON2 & 3.62 & 0.04 & NCEH1 & -3.73 & 0.00 \\
\hline CHN1 & 3.59 & 0.04 & AC010531.1 & -3.71 & 0.03 \\
\hline
\end{tabular}


Table 2. Top 20 disease/disorder conditions and their respectively associated DEGs based on disease network analysis of 218 DEGs between In-flight and Pre-flight.

\begin{tabular}{|c|c|}
\hline DISEASE/DISORDER & GENES \\
\hline Malignant Neoplasm of Breast & $\begin{array}{l}\text { THBS3, UBR4, ATF2, NBN, CRHR1, AREG, } \\
\text { HIF1A, PDPK1, COL7A1, ZNF404, BIRC2 }\end{array}$ \\
\hline Colorectal Carcinoma & $\begin{array}{l}\text { POSTN, SACS, FANCG, XAF1, ACSL4, INTS13, } \\
\text { COL7A1, NFATC1, C12ORF76, NDUFA1 }\end{array}$ \\
\hline Malignant Neoplasm of Prostate & $\begin{array}{l}\text { GREB1, HMGN5, SPON2, HIF1A, CRYL1, } \\
\text { CASZ1, ACSL4, NBN }\end{array}$ \\
\hline Prostatic Neoplasms & $\begin{array}{l}\text { CASZ1, NBN, GREB1, HIF1A, CRYL1, ACSL4, } \\
\text { SPON2, HMGN5 }\end{array}$ \\
\hline Schizophrenia & $\begin{array}{l}\text { PSAT1, BTBD9, CFAP65, CREB1, CCL2, } \\
\text { HSPA12A, DKK3, VRK2 }\end{array}$ \\
\hline Breast Carcinoma & $\begin{array}{l}\text { BIRC2, COL7A1, ZNF404, HIF1A, AREG, } \\
\text { CRHR1, PDPK1 }\end{array}$ \\
\hline Mammary Carcinoma, Human & $\begin{array}{l}\text { AREG, COL7A1, HIF1A, CRHR1, ZNF404, } \\
\text { PDPK1, BIRC2 }\end{array}$ \\
\hline Mammary Neoplasms & $\begin{array}{l}\text { AREG, COL7A1, PDPK1, ZNF404, CRHR1, } \\
\text { HIF 1A, BIRC2 }\end{array}$ \\
\hline Mammary Neoplasms, Human & $\begin{array}{l}\text { ZNF404, COL7A1, PDPK1, CRHR1, BIRC2, } \\
\text { HIF1A, AREG }\end{array}$ \\
\hline Unipolar Depression & CCL2, PEA15, HIF1A, CRHR1, CREB1, ACSLA \\
\hline Liver Cirrhosis, Experimental & GPT, TM6SF1, SGCB, ARHGAP9, CCL2 \\
\hline Major Depressive Disorder & HIF1A, CRHR1, PEA15, CCL2, CREB1 \\
\hline Non-small Cell Lung Carcinoma & E2F8, PSATI, AREG, HIF1A, COL7A1 \\
\hline Bipolar Disorder & HIF1A, CRHR1, CREB1, HMGXB4 \\
\hline Chemical And Drug Induced Liver Injury & HACL1, GPT, UBA5, CCL2 \\
\hline Chemical-induced Liver Toxicity & HACL1, UBA5, GPT, CCL2 \\
\hline Depressive Disorder & CREB1, CRHR1, DPP4, ACSL4 \\
\hline Disease Exacerbation & COL7A1, E2F8, ATF2, HIF1A \\
\hline Drug-induced Acute Liver Injury & GPT, HACL1, CCL2, UBA5 \\
\hline Drug-induced Liver Disease & HACL1, GPT, CCL2, UBA5 \\
\hline
\end{tabular}


Table 3. Gene pairs with significantly altered correlation coefficients between In-flight and Preflight (i.e., $\left.\left|\Delta \mathrm{CC}\left(\mathrm{CC}_{\text {In-flight }}-\mathrm{CC}_{\text {Pre-flight }}\right)\right|>0.70\right)$ in the top 11 signaling pathways identified from signaling pathway analysis of the 218 DEGs between In-flight and Pre-flight. Gene pairs with correlation coefficients changed from positive to less/more positive (Category 1), positive to negative (Category 2), negative to positive (Category 3) and negative to less/more negative (Category 4) were highlighted in blue, green, brown, and red, respectively.

\begin{tabular}{|c|c|c|c|}
\hline GENE PAIRS & CCPre-flight & CCIn-flight & $\Delta \mathrm{CC}$ \\
\hline \multicolumn{4}{|l|}{ Signal transduction } \\
\hline ARHGAP9-COLAA4 & -0.51 & 0.28 & 0.79 \\
\hline ARHGAP9 - THBS3 & 0.39 & -0.48 & -0.87 \\
\hline CCL2 - COLAA4 & -0.95 & -0.07 & 0.88 \\
\hline CCL2 - CREB1 & 0.93 & -0.09 & -1.02 \\
\hline CCL2 - CRHR1 & 0.68 & -0.11 & -0.79 \\
\hline CCL2 - THBS3 & 0.68 & -0.19 & -0.87 \\
\hline CCNC-COLAA4 & 0.46 & -0.65 & -1.11 \\
\hline CCNC-CRHRI & -0.58 & 0.65 & 1.23 \\
\hline$C C N C-T H B S 3$ & -0.41 & 0.34 & 0.75 \\
\hline CHN1 - CRHR1 & 0.43 & -0.59 & -1.02 \\
\hline CHN1 - HIF1A & -0.36 & 0.34 & 0.70 \\
\hline COLAA4 - CREB1 & -0.93 & -0.03 & 0.90 \\
\hline COLAA4 - NFATC1 & -0.77 & 0.50 & 1.27 \\
\hline COLAA4 - PDPK1 & 0.41 & -0.50 & -0.91 \\
\hline CREB1 - CRHR1 & 0.80 & -0.03 & -0.83 \\
\hline CREB1 - THBS3 & 0.79 & 0.05 & -0.74 \\
\hline CRHR1 - KIDINS220 & 0.59 & -0.14 & -0.73 \\
\hline CRHR1 - NFATC1 & 0.83 & -0.47 & -1.30 \\
\hline CRHRI - PDPK1 & -0.57 & 0.34 & 0.91 \\
\hline CRHR1 - YES1 & 0.40 & -0.71 & -1.11 \\
\hline CTNNBIP1 - NFATC1 & 0.07 & 0.85 & 0.78 \\
\hline KIDINS220 - PDPK1 & -0.74 & 0.30 & 1.04 \\
\hline KIDINS220 - SOS2 & -0.86 & 0.29 & 1.15 \\
\hline KIDINS220 - YES1 & 0.70 & 0.00 & -0.70 \\
\hline NFATC1 - THBS3 & 0.72 & -0.34 & -1.06 \\
\hline$P D P K 1-S O S 2$ & 0.63 & -0.22 & -0.85 \\
\hline SOS2 - YES1 & -0.61 & 0.17 & 0.78 \\
\hline \multicolumn{4}{|l|}{ Immune system } \\
\hline ATF2 - NFATC1 & -0.03 & -0.75 & -0.72 \\
\hline$B I R C 2-P D P K 1$ & -0.73 & 0.13 & 0.86 \\
\hline CREB1 - EIF4E2 & -0.94 & 0.07 & 1.01 \\
\hline CREB1 - IL7 & 0.91 & -0.03 & -0.94 \\
\hline CREB1 - UBR4 & 0.90 & -0.28 & -1.18 \\
\hline CREB1 - XAF1 & 0.71 & -0.03 & -0.74 \\
\hline EIF4E2 - IL7 & -0.87 & 0.10 & 0.97 \\
\hline
\end{tabular}




\begin{tabular}{|c|c|c|c|}
\hline EIF4E2 - NFATC1 & -0.87 & 0.46 & 1.33 \\
\hline EIF4E2 - UBR4 & -0.95 & 0.40 & 1.35 \\
\hline EIF4E2 - XAF1 & -0.72 & 0.10 & 0.82 \\
\hline IL7 - UBR4 & 0.88 & -0.34 & -1.22 \\
\hline NFATC1 - UBR4 & 0.85 & -0.18 & -1.03 \\
\hline PDPK1 - UBR4 & -0.55 & 0.34 & 0.89 \\
\hline$U B A 5-U B R 4$ & 0.59 & -0.27 & -0.86 \\
\hline UBR4 - XAF1 & 0.88 & -0.16 & -1.04 \\
\hline UBR4 - YES1 & 0.35 & -0.59 & -0.94 \\
\hline \multicolumn{4}{|l|}{ Gene expression } \\
\hline$A A R S 2-Z N F 253$ & -0.57 & 0.23 & 0.80 \\
\hline AARS2 - ZNF606 & -0.91 & 0.21 & 1.12 \\
\hline$A A R S 2-Z N F 711$ & -0.60 & 0.31 & 0.91 \\
\hline RRN3 - ZNF184 & -0.51 & 0.34 & 0.85 \\
\hline RRN3 - ZNF606 & -0.06 & 0.87 & 0.93 \\
\hline RRN3 - ZNF711 & -0.22 & 0.50 & 0.72 \\
\hline \multicolumn{4}{|l|}{ Metabolism } \\
\hline$A C S L 4-A R S K$ & 0.56 & -0.66 & -1.22 \\
\hline ACSL4-CCNC & 0.75 & -0.74 & -1.49 \\
\hline$A C S L 4-G P T$ & -0.66 & 0.85 & 1.61 \\
\hline ACSL4 - NDUFAI & 0.81 & 0.05 & -0.76 \\
\hline ACSL4 - PIKFYVE & -0.22 & 0.69 & 0.91 \\
\hline$A R S K-G M 2 A$ & 0.48 & -0.34 & -0.82 \\
\hline$C C N C-G M 2 A$ & 0.65 & -0.44 & -1.09 \\
\hline$C C N C-P S A T 1$ & 0.88 & -0.23 & -1.11 \\
\hline GPT-NDUFAI & -0.59 & 0.24 & 0.83 \\
\hline GPT-PIKFYVE & -0.05 & 0.79 & 0.84 \\
\hline GPT-PSAT1 & -0.65 & 0.46 & 1.11 \\
\hline \multicolumn{4}{|c|}{ Metabolism of proteins } \\
\hline$A R S K-D P P 4$ & -0.27 & 0.57 & 0.84 \\
\hline CCL2 - DPP4 & 0.79 & -0.26 & -1.05 \\
\hline CCL2 - PCSK1 & 0.90 & -0.16 & -1.06 \\
\hline CCL2 - SPON2 & 0.77 & -0.03 & -0.80 \\
\hline$D P P 4-G N E$ & 0.74 & -0.21 & -0.95 \\
\hline DPP4 - MAGT1 & -0.57 & 0.37 & 0.94 \\
\hline MAGT1 - PCSK1 & -0.85 & 0.21 & 1.06 \\
\hline PCSK1 - SPON2 & 0.84 & 0.01 & -0.83 \\
\hline \multicolumn{4}{|c|}{ Developmental biology } \\
\hline CCNC-COLAA4 & 0.46 & -0.65 & -1.11 \\
\hline$C L T B-S C N 2 B$ & -0.39 & 0.37 & 0.76 \\
\hline COLAA4 - CREB1 & -0.93 & -0.03 & 0.90 \\
\hline SOS2 - YES1 & -0.61 & 0.17 & 0.78 \\
\hline \multicolumn{4}{|c|}{ Metabolism of lipids and lipoproteins } \\
\hline$A C S L 4-A R S K$ & 0.56 & -0.66 & -1.22 \\
\hline
\end{tabular}


bioRxiv preprint doi: https://doi.org/10.1101/2022.02.06.479323; this version posted February 7,2022 . The copyright holder for this preprint (which was not certified by peer review) is the author/funder. All rights reserved. No reuse allowed without permission.

\begin{tabular}{|c|c|c|c|}
\hline ACSL4 - CCNC & 0.75 & -0.74 & -1.49 \\
\hline ACSL4 - PIKFYVE & -0.22 & 0.69 & 0.91 \\
\hline$A R S K-G M 2 A$ & 0.48 & -0.34 & -0.82 \\
\hline$C C N C-G M 2 A$ & 0.65 & -0.44 & -1.09 \\
\hline \multicolumn{4}{|l|}{ Axon guidance } \\
\hline$C L T B-S C N 2 B$ & -0.39 & 0.37 & 0.76 \\
\hline COLAA4 - CREB1 & -0.93 & -0.03 & 0.90 \\
\hline SOS2 - YES1 & -0.61 & 0.17 & 0.78 \\
\hline \multicolumn{4}{|c|}{ Innate immune system } \\
\hline ATF2 - NFATC1 & -0.03 & -0.75 & -0.72 \\
\hline BIRC2 - PDPK1 & -0.73 & 0.13 & 0.86 \\
\hline \multicolumn{4}{|l|}{ Disease } \\
\hline CCNC-CHMP4C & 0.60 & -0.38 & -0.98 \\
\hline CHMP $4 C-C R E B 1$ & -0.85 & 0.31 & 1.16 \\
\hline CHMP $4 C-P D P K 1$ & 0.44 & -0.63 & -1.07 \\
\hline
\end{tabular}




\section{Figure legends}

Figure 1. Gene pair correlations of the whole genome (30645 transcripts for 23115 genes) for 10 astronauts during Pre-flight (A), In-flight (B) and Post-flight (C). Positive and negative correlations are represented by blue and red, respectively.

Figure 2. Gene pair correlations (1: Pre-flight, 2: In-flight, and 3: Post-flight) and differentials of gene pair correlations (4: between In-flight and Pre-flight, and 5: between Post-flight and In-flight) for the differentially expressed genes (DEGs) in the top 11 signaling pathways: signal transduction (A), immune system $(\mathbf{B})$, gene expression $(\mathbf{C})$, metabolism (D), metabolism of proteins $(\mathbf{E})$, generic transcription pathway (F), developmental biology (G), metabolism of lipids and lipoproteins $(\mathbf{H})$, axon guidance (I), innate immune system $(\mathbf{J})$ and disease $(\mathbf{K})$. The top 11 signaling pathways were identified from InnateDB analysis of the 218 DEGs $\left(\left|\log _{2} \mathrm{FC}\right| \geq 3.00\right.$ and $p<0.05$ ). Positive and negative gene pair correlations are represented in blue and red, respectively; and positive and negative differentials of gene pair correlations are represented in green and red, respectively.

Figure 3. Protein-protein interaction (A), disease-gene (B), drug-gene (C) and miRNA-gene (D) networks for the 218 differentially expressed genes (DEGs) between Pre-flight and In-flight gene expression datasets collected from 10 astronauts ( 8 males and 2 females). Nodes with higher degrees of connectivity were shown in larger size and darker color. Genes identified for the rapid $\mathrm{RT}^{2}$-PCR assay kit were labelled in the networks. Genes CCNC, CTNNBIP1, EIF4E2 and RRN3 are not present in the disease-gene network; genes BIRC2, CCNC, CHMP4C, CTNNBIP1, EIF4E2, IL7, KIDINS220, MAGT1, PIKFYVE, RRN3, SOS2, SPON2, THBS3, XAF1 and ZNF606 are not present in the drug-gene network; and genes CRHR1, DPP4, GPT and SPON2 are not present in the miRNA-gene network, respectively. 
Figure 1

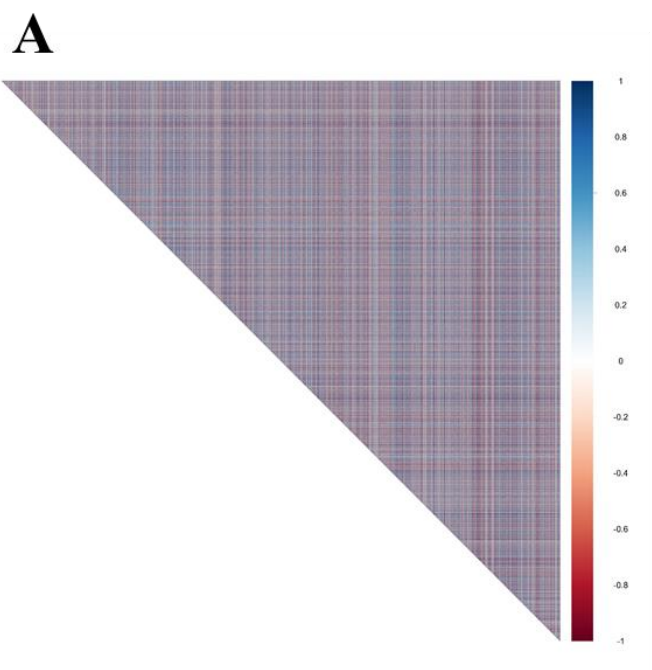

Pre-flight
B

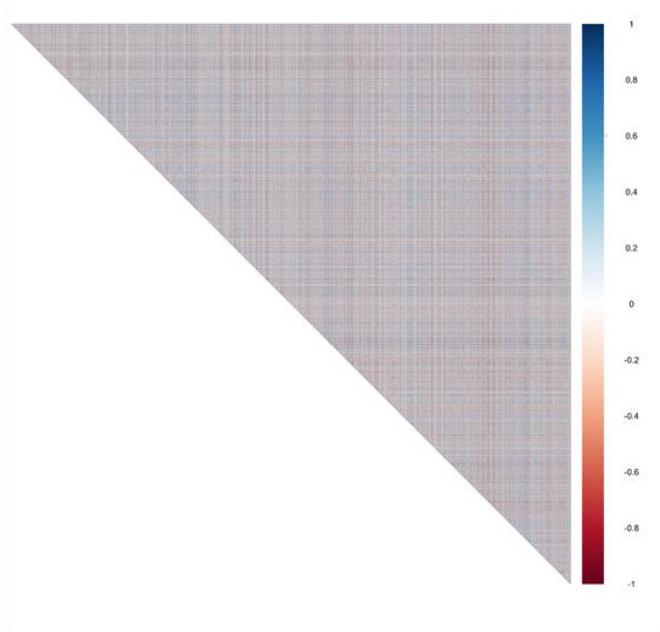

In-flight

\section{C}

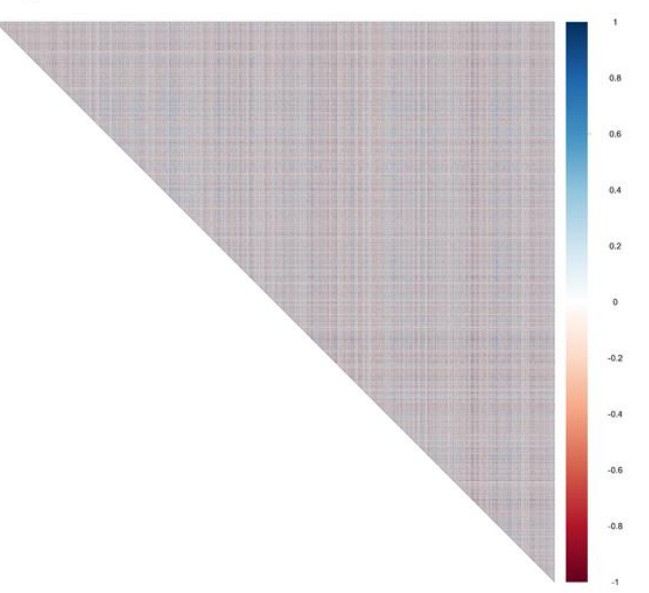

Post-flight 
Figure 2

Figure 2A

\section{Signal transduction}

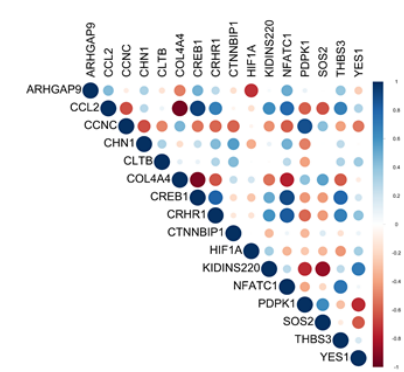

1

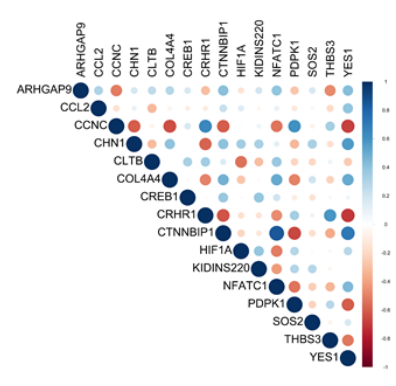

2

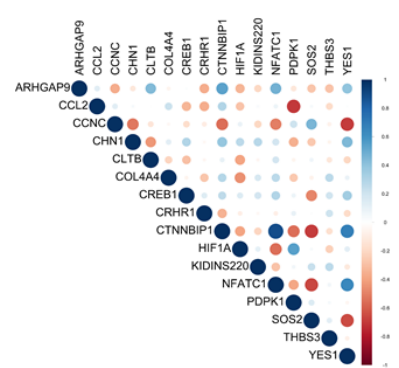

3

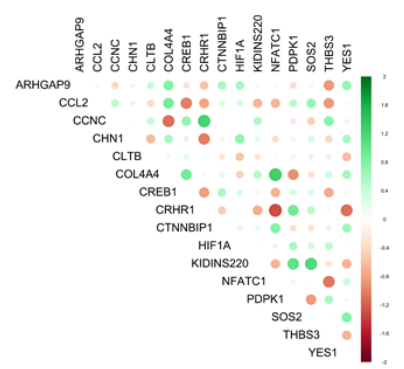

4

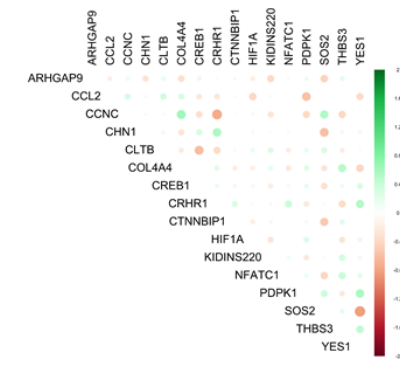

5

Figure 2B

\section{Immune system}

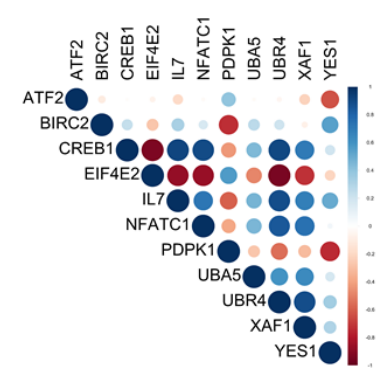

1

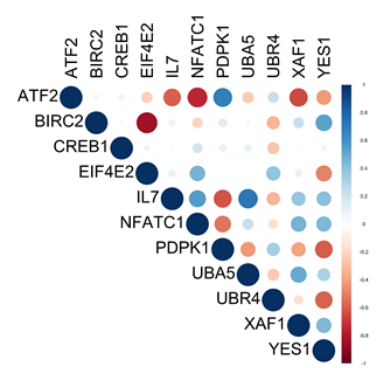

2

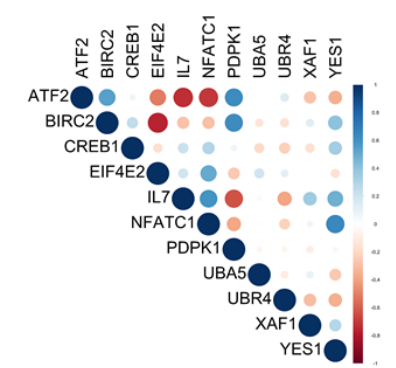

3

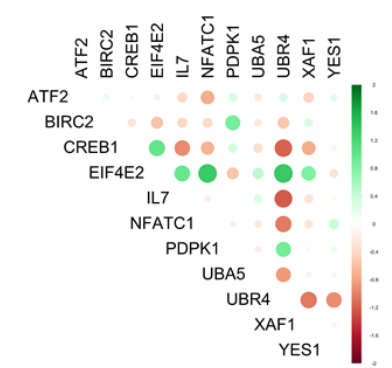

4

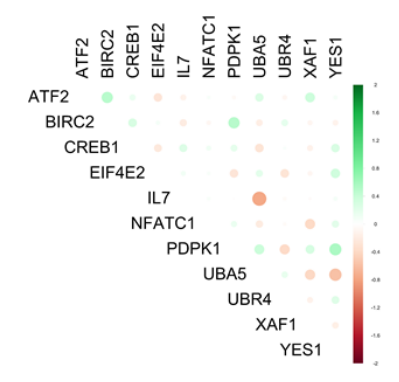

5 
Figure 2C

\section{Gene expression}

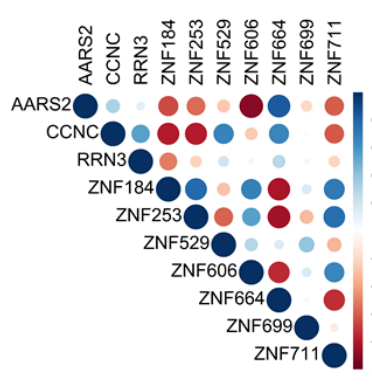

1

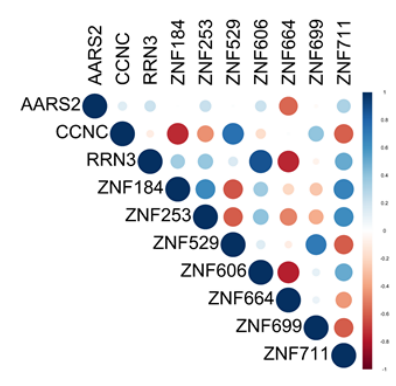

2

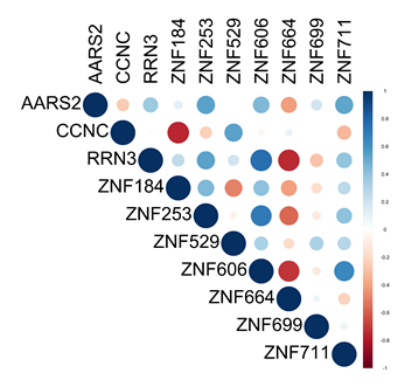

3

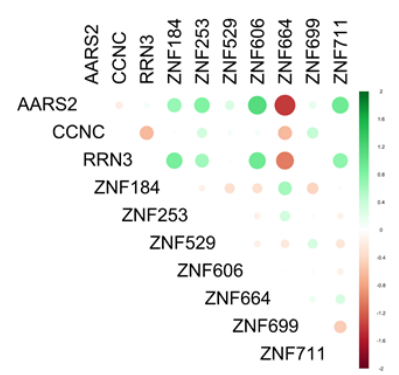

4

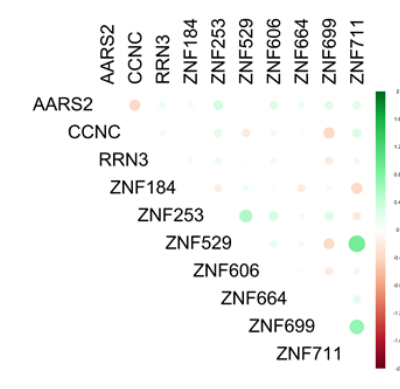

5

Figure 2D

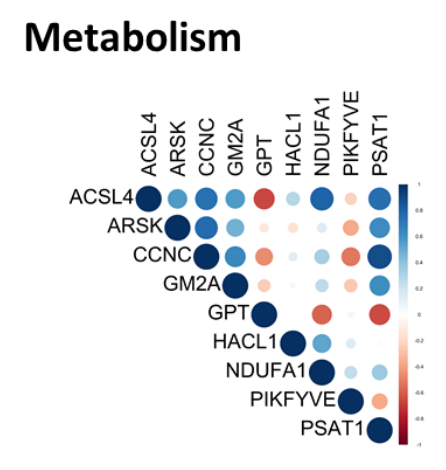

1

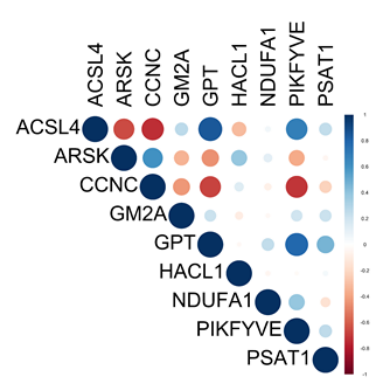

2

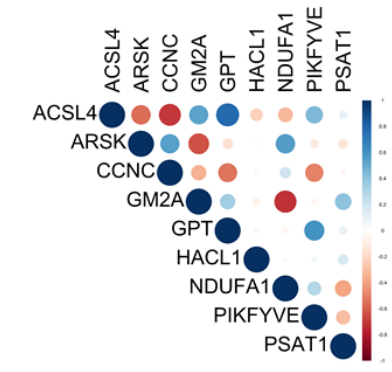

3

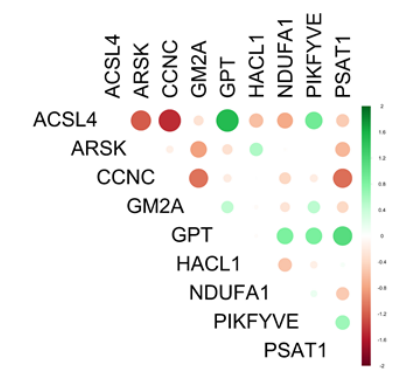

4

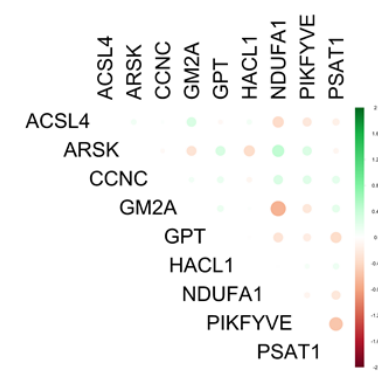

5 
Figure 2E

\section{Metabolism of proteins}

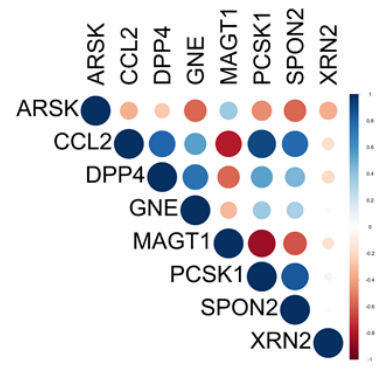

1

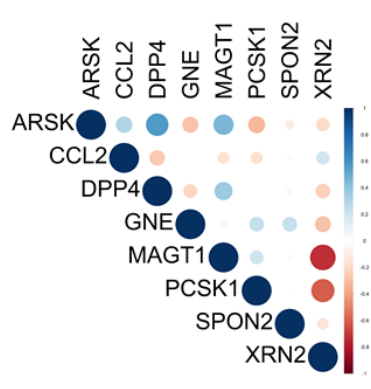

2

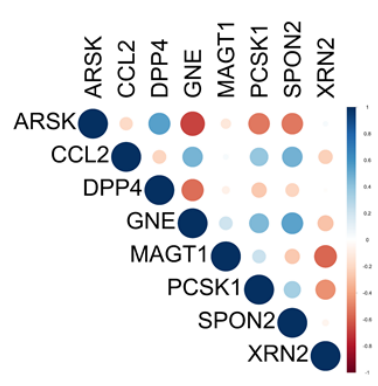

3

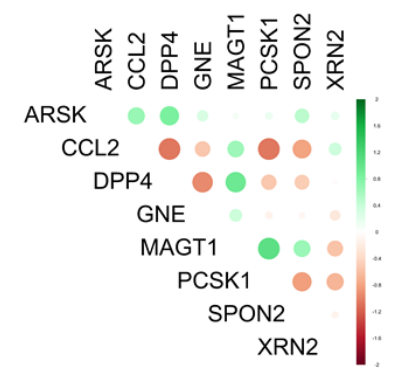

4

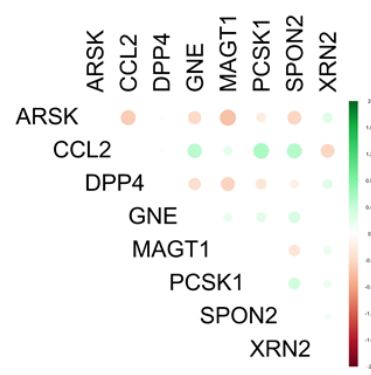

5

Figure 2F

\section{Generic transcription pathway}

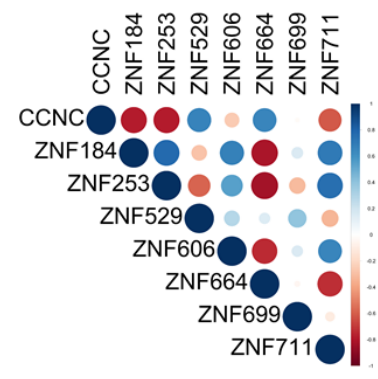

1

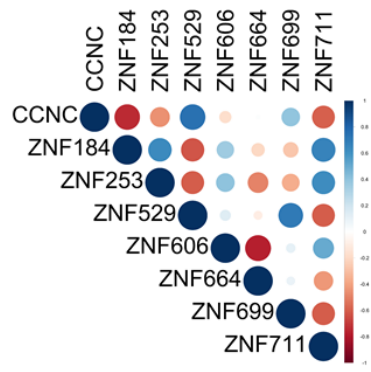

2

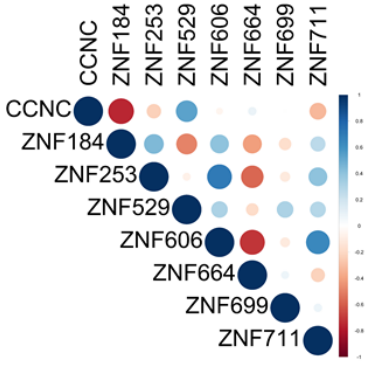

3

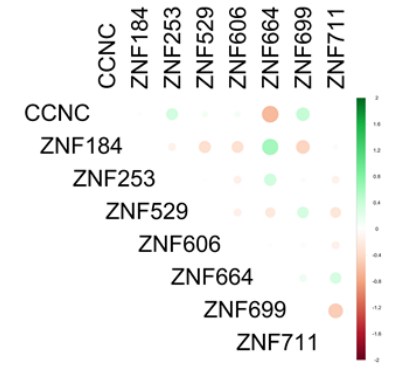

4

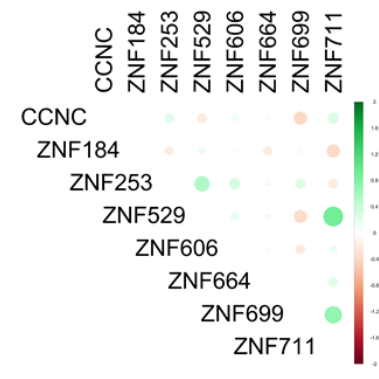

5 
Figure 2G

\section{Developmental biology}

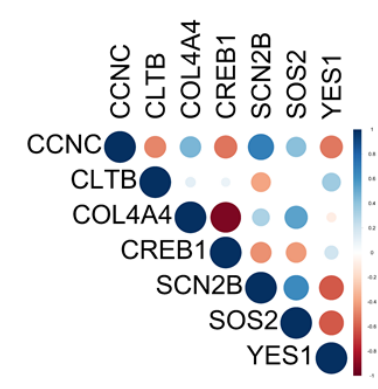

1

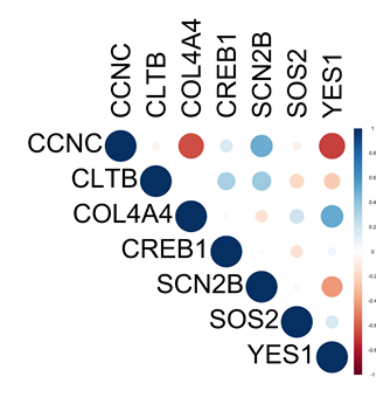

2

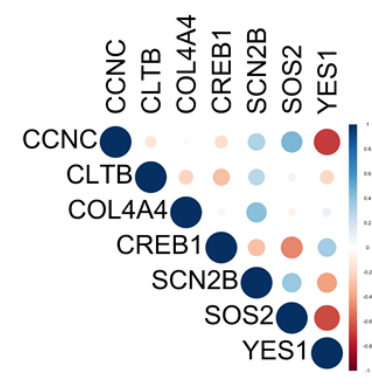

3

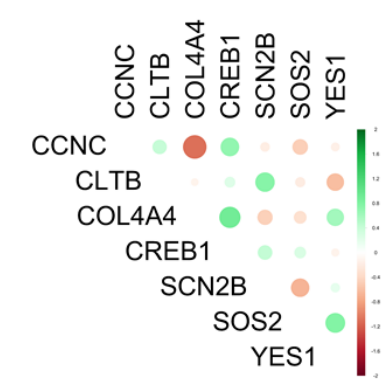

4

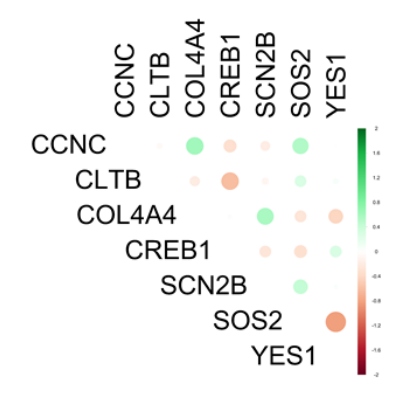

5

Figure $2 \mathrm{H}$

\section{Metabolism of lipids and lipoproteins}

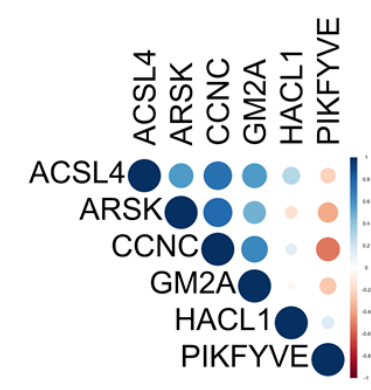

1

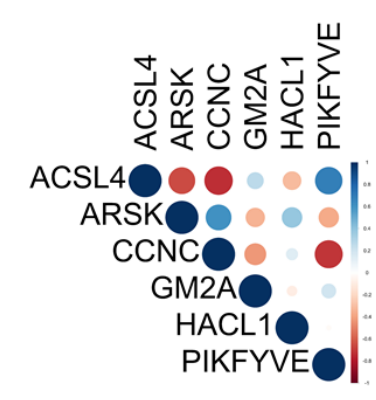

2

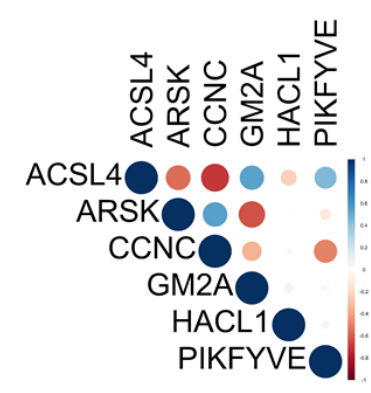

3

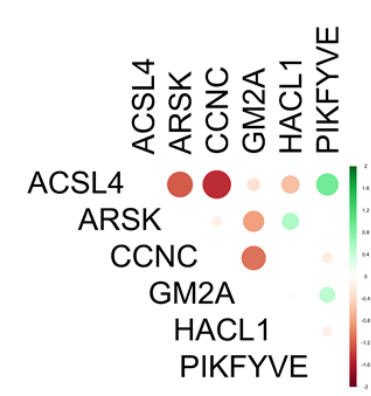

4

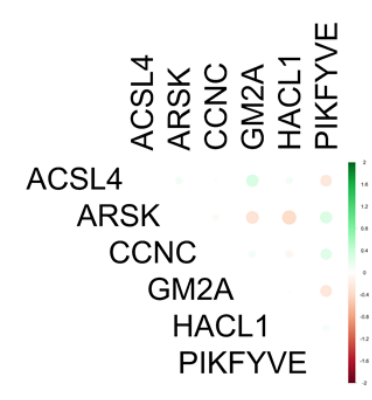

5 
Figure 2I

\section{Axon guidance}

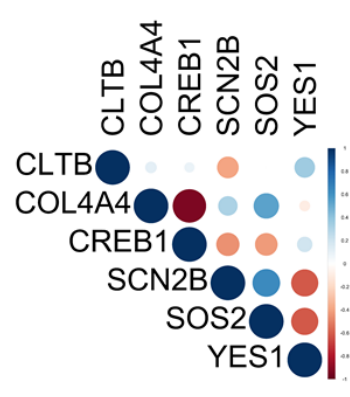

1

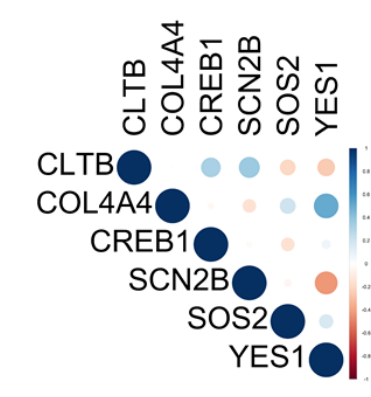

2

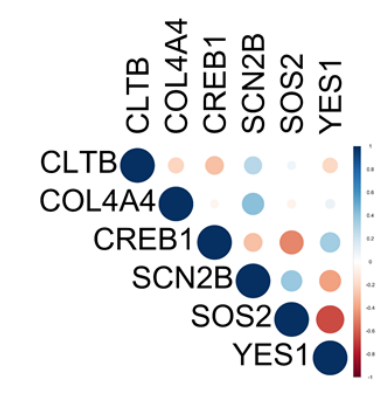

3

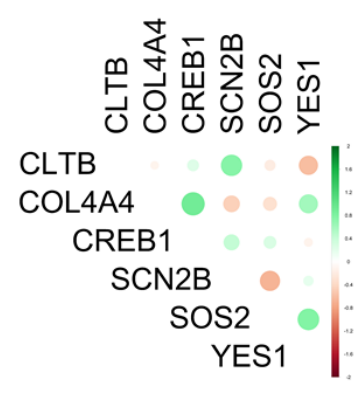

4

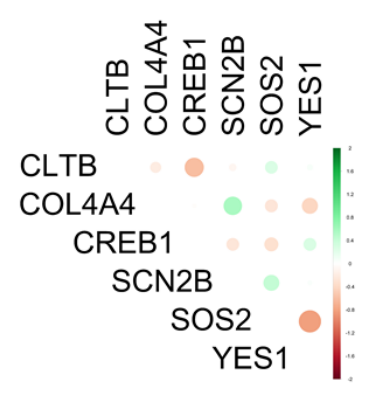

5

Figure 2J

\section{Innate immune system}

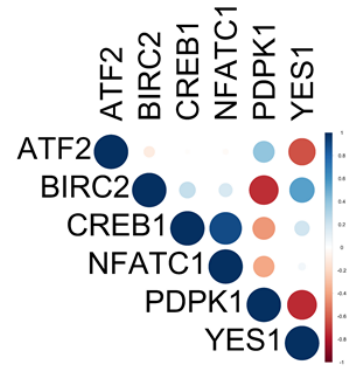

1

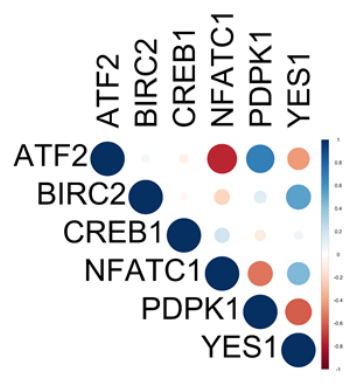

2

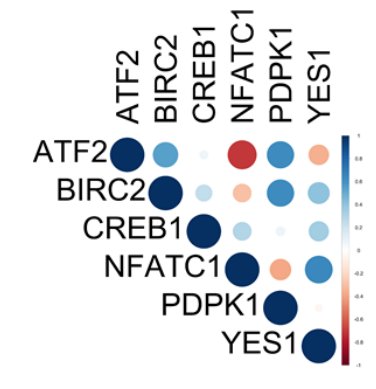

3

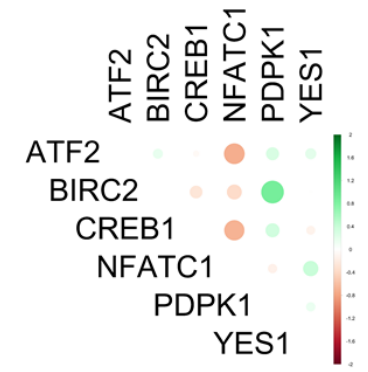

4

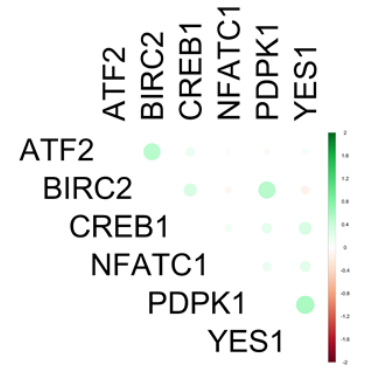

5 
Figure 2K

\section{Disease

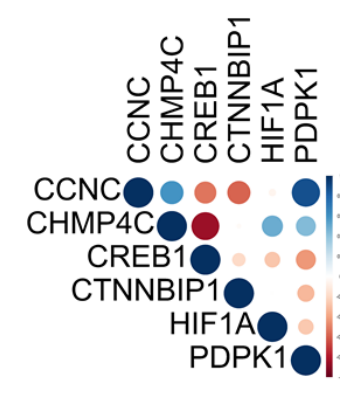

1

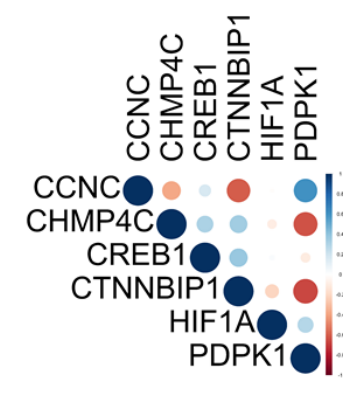

2

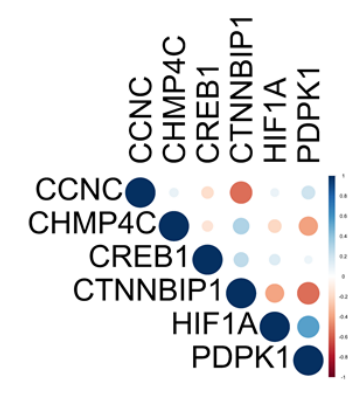

3

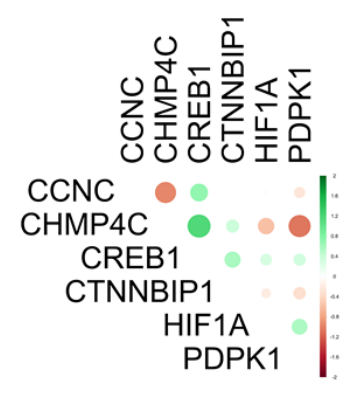

4

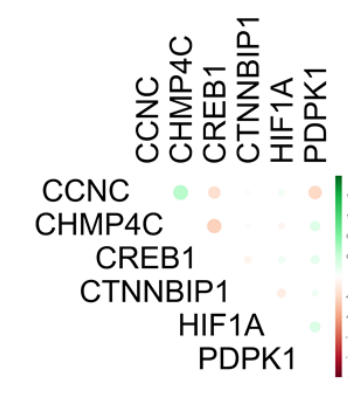

5 


\section{Figure 3}

Figure 3A

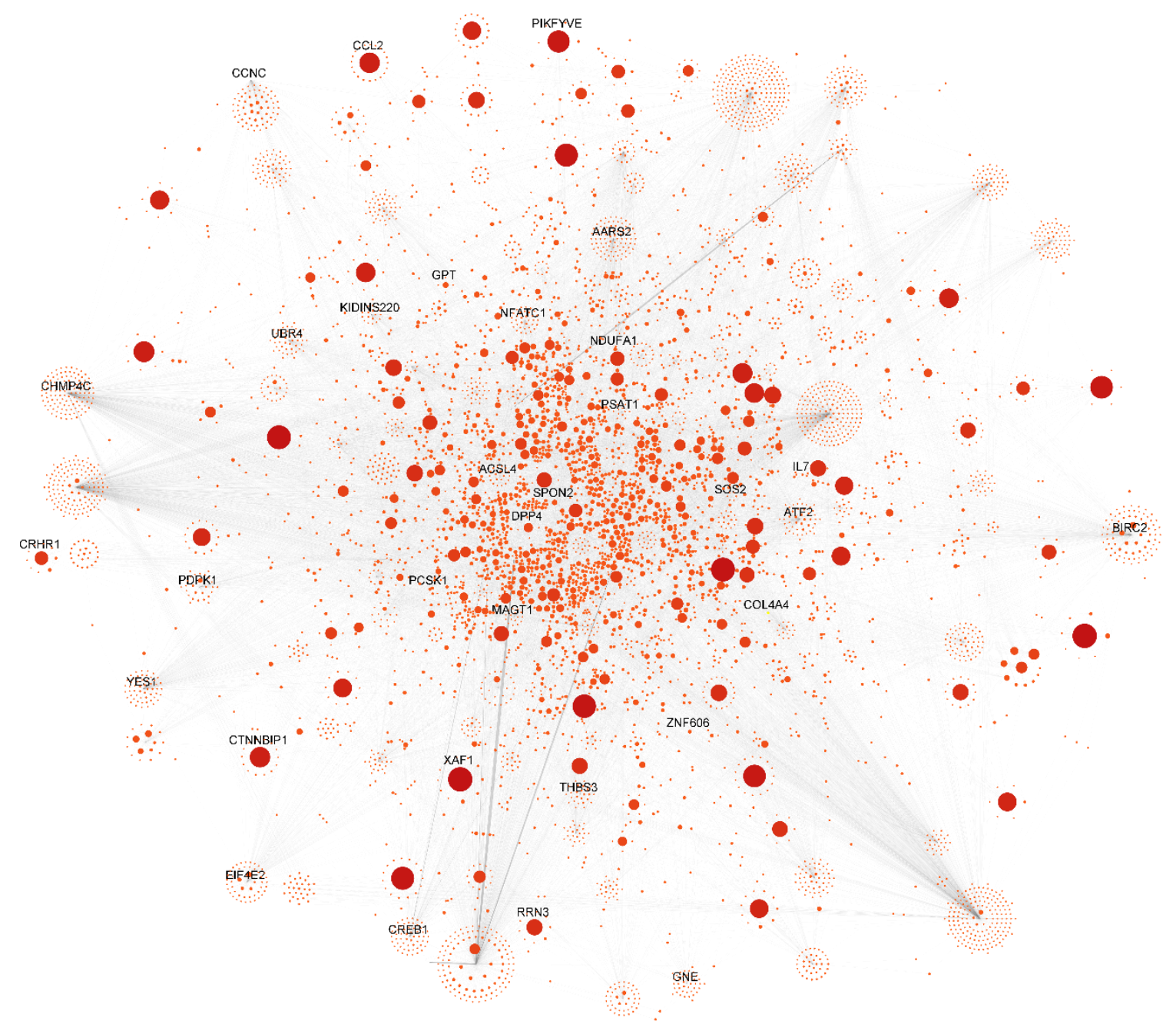


Figure 3B

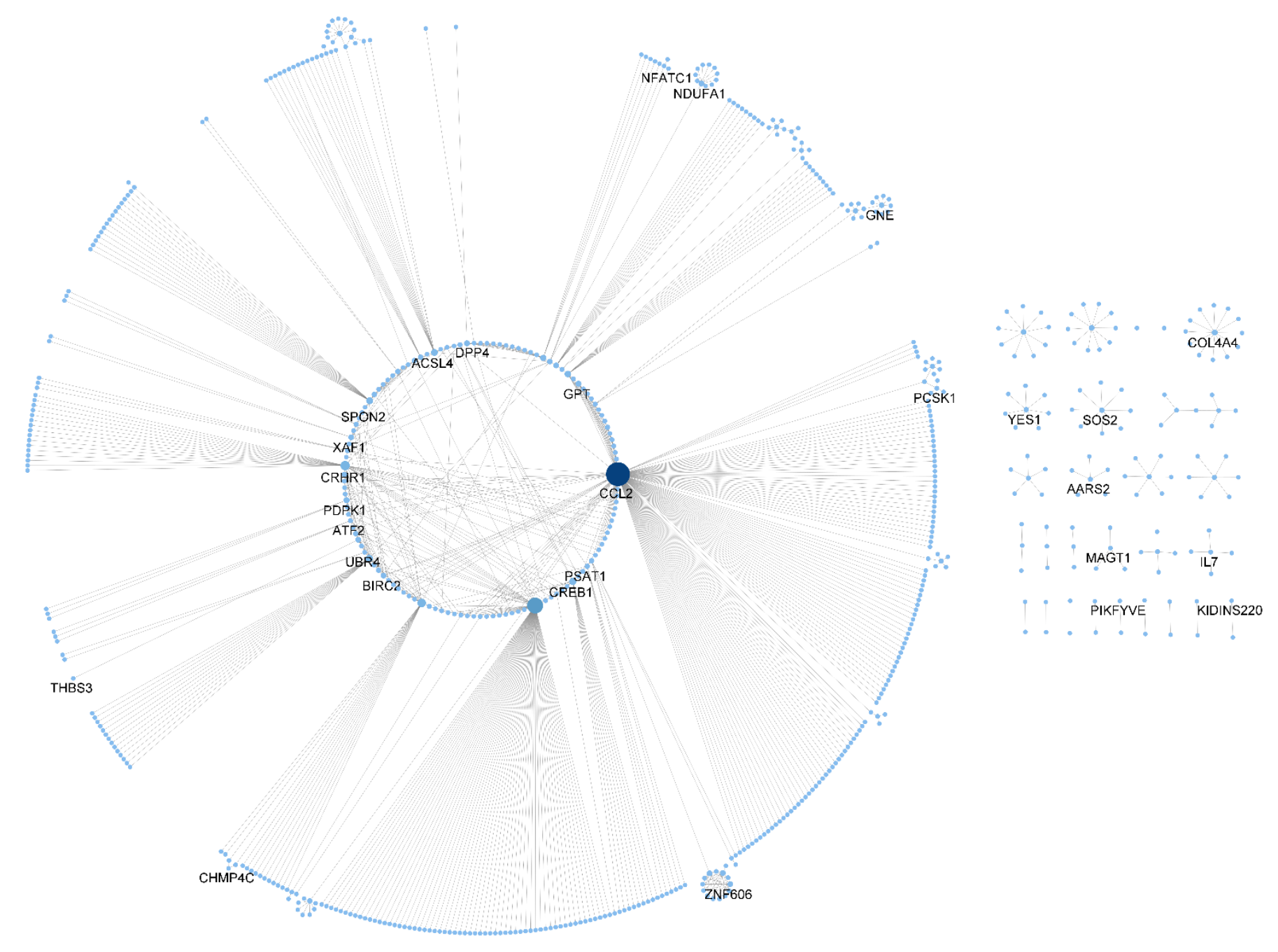


Figure 3C
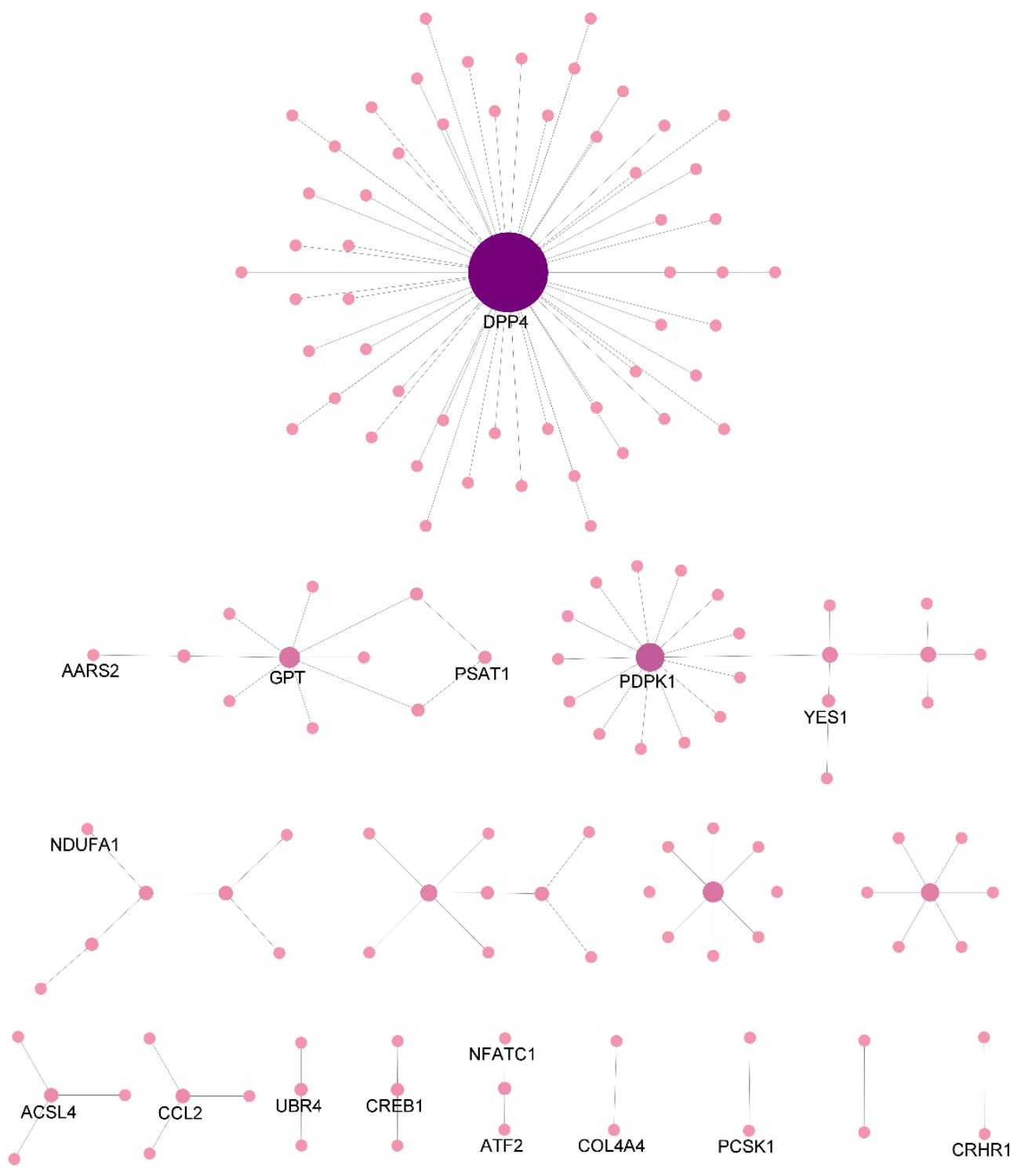
Figure 3D

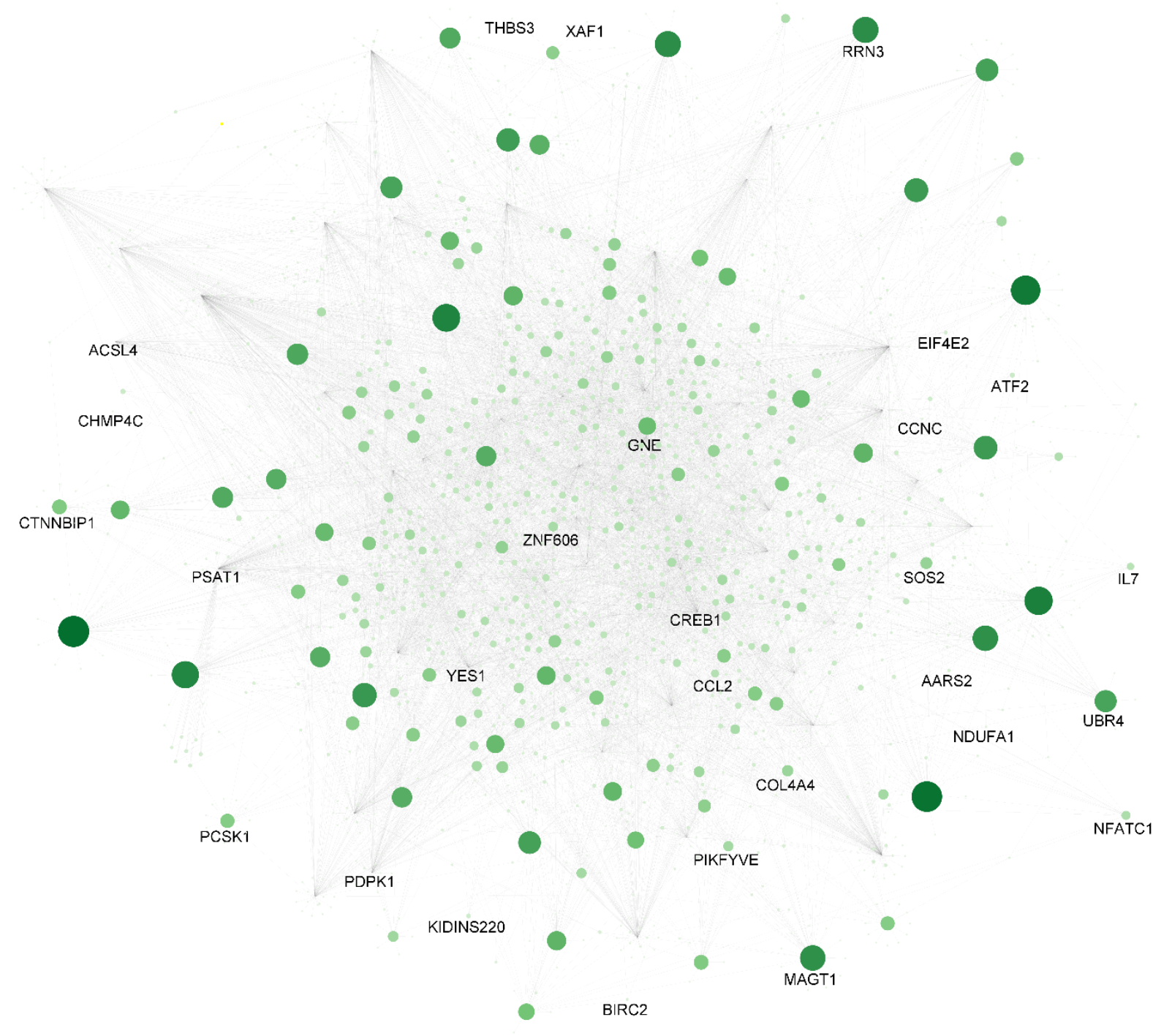

